\title{
Geometric series with randomly increasing exponents
}

\author{
J. Neunhäuserer \\ Reitstallweg 9, D-38640 Goslar, Germany \\ neunchen@aol.com
}

\begin{abstract}
We prove results on absolute continuity and singularity of the distribution of geometric series with randomly increasing exponents.

MSC 2010: Primary 26A46, 26A30, Secondary 28A78, 28A80

Key-words: random geometric series, singularity, absolute continuity, Hausdorff dimension, density
\end{abstract}

\section{Introduction}

There are two techniques in geometric measure theory which were successfully applied in the last decades. On the one hand we have results on the Hausdorff dimension of geometric measures, which especially imply their singularity. On the other hand transversality properties of a symbolic coding allow to prove generic results on absolute continuity and density of geometric measures. We especially have a couple of results on absolute continuity of the distribution of power series with random coefficients $[14,15,13,19,17,1]$ and other non-uniform self similar measures [11, 12, 9], as well as results on geometric Markov measures [6].

We change the focus here to geometric series with randomly increasing exponents. These random variables and their distribution are natural and worth to study. Nevertheless we find no results in the literature.

In this paper we determine the border of singularity and generic absolute continuity of the distribution of a geometric series with exponents chosen according to a random variable $X$ by $e^{-H(X) / E(X)}$, see Theorem 2.1 below. We calculate this quantity for some well known distributions $X$. The singularity assertion follows from an estimate on the Hausdorff dimension of the distribution, see Theorem 3.1. The proof of this result uses local dimension, Shannon's entropy theorem and the law of large numbers. In Theorem 4.1 we determine intervals, where the distribution of geometric series with random exponents have generically a density in $L^{q}$. The assertion of absolute continuity of the measures is just a corollary to this theorem. The proof is based on the transversality of the coding map and the local density of the distribution. The transversality techniques give an upper bound on the interval absolute continuity, which we conjecture not to be sharp. 


\section{Definition of measures and main results}

Let $X$ be a random variable taking values in $\mathbb{N}$ and set $p_{i}:=P(X=i)$ for $i \in \mathbb{N}$. We assume that $X$ has finite expectation value

$$
E(X)=\sum_{i \in \mathbb{N}} i p_{i}<\infty
$$

and entropy

$$
H(X)=-\sum_{i \in \mathbb{N}} p_{i} \log \left(p_{i}\right)<\infty .
$$

Here we use the convention $p_{i} \log \left(p_{i}\right)=0$ for $p_{i}=0$.

For $\beta \in(0,1)$ we consider geometric series with randomly increasing exponents,

$$
X_{\beta}=\sum_{k=1}^{\infty} \beta^{X_{1}+X_{2}+\cdots+X_{k}},
$$

where the random variables $X_{k}$ are independent and identically distributed according to $X$. Let $\mu_{\beta}$ be the Borel probability measure on $\mathbb{R}$ describing the distribution of $X_{\beta}$, that is

$$
\mu_{\beta}(B)=P\left(X_{\beta} \in B\right)
$$

for all Borel sets $B \subseteq \mathbb{R}$. Now we introduce another description of $\mu_{\beta}$. Consider $\mathbb{N}^{\mathbb{N}}$ equipped with the product of the discrete topology on $\mathbb{N}$. This is a perfect, totally disconnected Polish space, see [3]. The random variable $X$ induces a product measure $\mu$ on $\mathbb{N}^{\mathbb{N}}$ with

$$
\mu\left(\left[n_{1}, n_{2} \ldots, n_{k}\right]\right)=p_{n_{1}} \cdot p_{n_{2}} \cdots p_{n_{k}}
$$

for cylinder sets $\left[n_{1}, n_{2} \ldots, n_{k}\right]$ in $\mathbb{N}^{\mathbb{N}}$. Obviously the distribution of a geometric series with randomly increasing exponents $\mu_{\beta}$ is the projection of $\mu$ under the map $\pi_{\beta}: \mathbb{N}^{\mathbb{N}} \longmapsto \mathbb{R}$ given by

$$
\pi_{\beta}\left(\left(n_{k}\right)\right)=\sum_{k=1}^{\infty} \beta^{n_{1}+n_{2}+\cdots+n_{k}} .
$$

Using the description $\mu_{\beta}=\pi_{\beta}(\mu)=\mu \circ \pi_{\beta}^{-1}$, a simple calculation shows that $\mu_{\beta}$ is selfsimilar with respect to the iterated function system $\left\{T_{i} x=\beta^{i}(x+1) \mid i \in \mathbb{N}\right\}$. That means

$$
\mu_{\beta}=\sum_{i \in \mathbb{N}} p_{i} T_{i}\left(\mu_{\beta}\right),
$$

see [8] for the general theory of iterated function systems.

Recall that $\mu_{\beta}$ is singular with respect to the Lebesgue measure $\lambda$ if there is a Borel set $B \subseteq \mathbb{R}$ with $\lambda(B)=0$ and $\mu_{\beta}(B)=1$. Our $\mu_{\beta}$ is absolutely continuous, if $\lambda(B)=0$ implies $\mu_{\beta}(B)=0$ for all Borel sets $B \subseteq \mathbb{R}$. Now we are prepared to state the main result of this paper. 
Theorem 2.1 For all $\beta \in(0,1)$ with

$$
\beta<e^{-H(X) / E(X)},
$$

the distribution $\mu_{\beta}$ of a power series with randomly increasing exponents $X_{\beta}$ is singular with respect to the Lebesgue measure. On the other hand for $\lambda$-almost all

$$
\beta \in\left(e^{-H(X) / E(X)}, 0.668\right)
$$

the measure $\mu_{\beta}$ is absolutely continuous with respect to the Lebesgue measure.

The upper bound 0.668 in our theorem is due to transversality techniques we use in section 4. We conjecture that this bound is not sharp. It would be natural to have one here, but we have no idea to prove this.

We now determine the boundary $\mathfrak{b}(X)=e^{-H(X) / E(X)}$ of generic absolute continuity of $X_{\beta}$ for some classical distributions $X$. In the following, numerical values are truncated to three decimals.

First consider the uniform distribution on $\{1, \ldots, n\}$. We obviously have

$$
\begin{gathered}
\left(\mathfrak{b}\left(X_{n}\right)\right)=\left(\sqrt[n+1]{1 / n^{2}}\right) \\
=(1,0.630,0.577,0.574,0.585,0.599,0.614,0.629,0.644,0.658,0.670, \ldots)
\end{gathered}
$$

For the symmetric Binomial distribution $X_{n}$ on $\{1, \ldots, n\}$, given by

$$
P\left(X_{n}=i\right)=\left(\begin{array}{c}
n-1 \\
i-1
\end{array}\right) 2^{-n+1}
$$

we get by some computation

$$
\begin{gathered}
\left(\mathfrak{b}\left(X_{n}\right)\right)=\left(\exp \left(\frac{2}{n+1} \sum_{i=1}^{n}\left(\begin{array}{c}
n-1 \\
i-1
\end{array}\right) 2^{-n+1} \log \left(\left(\begin{array}{c}
n-1 \\
i-1
\end{array}\right) 2^{-n+1}\right)\right)\right) \\
=(1,0.636,0.595,0.605,0.625,0.647,0.667,0.686, \ldots)
\end{gathered}
$$

For the geometric distribution on $\mathbb{N}$, given by $P\left(X_{p}=i\right)=(1-p)^{i-1} p$, we have

$$
\mathfrak{b}\left(X_{p}\right)=p^{p}(1-p)^{1-p}
$$

This result can also be deduced from Theorem 4.1 of Peres and Solomyak on biased Bernoulli convolutions [15]. Now consider the Poisson distribution $X_{\mu}$ on $\mathbb{N}$ with expectation value $\mu \in \mathbb{R}^{+}$, given by

$$
P\left(X_{\mu}=i\right)=\frac{(\mu-1)^{i-1}}{(i-1) !} e^{-\mu+1} .
$$


We have

$$
\mathfrak{b}\left(X_{\mu}\right)=\exp \left(\frac{1}{\mu} \sum_{i=1}^{\infty} \frac{(\mu-1)^{i-1}}{(i-1) !} e^{-\mu+1} \log \left(\frac{(\mu-1)^{i-1}}{(i-1) !} e^{-\mu+1}\right) .\right.
$$

For $\mu=m \in \mathbb{N}$ we obtain by some computation,

$$
\mathfrak{b}\left(X_{m}\right)=(1,0.521,0.566,0.617,0.658,0.692, \ldots)
$$

We see that Theorem 2.1 gives intervals of absolute continuity for classical distributions, although the upper bound on absolute continuity in the result is not expected to be sharp.

\section{Dimension estimates and singularity}

We apply the dimension theory of geometric measures in order to obtain the assertion of singularity in Theorem 2.1. For $\beta \in(0,1)$ we define a metric on $\mathbb{N}^{\mathbb{N}}$ by

$$
d_{\beta}\left(\left(n_{k}\right),\left(m_{k}\right)\right)=\beta^{n_{1}+n_{2}+\cdots+n_{i\left[\left(n_{k}\right),\left(m_{k}\right)\right]}}
$$

where

$$
i\left[\left(n_{k}\right),\left(m_{k}\right)\right]=\max \left\{k \mid n_{k}=m_{k}\right\} .
$$

In the following $B_{\epsilon}^{\beta}$ denotes the $\epsilon$-ball and $|A|_{\beta}$ denotes the diameter in the metric space $\left(\mathbb{N}^{\mathbb{N}}, d_{\beta}\right)$. This metric is adapted to the coding map $\pi_{\beta}$, defined in the last section, in the following sense:

Lemma $3.1 \pi_{\beta}$ is Lipschitz continuous on $\left(\mathbb{N}^{\mathbb{N}}, d_{\beta}\right)$.

Proof. We have

$$
\begin{gathered}
d_{\beta}\left(\pi_{\beta}\left(n_{k}\right), \pi_{\beta}\left(m_{k}\right)\right)=\left|\sum_{k=1}^{\infty} \beta^{n_{1}+n_{2}+\cdots+n_{k}}-\beta^{m_{1}+m_{2}+\cdots+m_{k}}\right| \\
=\beta^{n_{1}+n_{2}+\cdots+n_{i\left[\left(n_{k}\right),\left(m_{k}\right)\right]} \mid} \sum_{k=i\left[\left(n_{k}\right),\left(m_{k}\right)\right]+1}^{\infty} \beta^{n_{1}+n_{2}+\cdots+n_{k}}-\beta^{m_{1}+m_{2}+\cdots+m_{k}} \mid \\
\leq d_{\beta}\left(\left(n_{k}\right),\left(m_{k}\right)\right) \sum_{k=1}^{\infty} \beta^{k}=\frac{\beta}{1-\beta} d_{\beta}\left(\left(n_{k}\right),\left(m_{k}\right)\right) .
\end{gathered}
$$

We are able to calculate the local dimension of the product measure $\mu$ on $\mathbb{N}^{\mathbb{N}}$ with respect to the metric $d_{\beta}$. 
Proposition 3.1 The local dimension of $\mu$ is given by

$$
\operatorname{dim}\left(\mu,\left(n_{k}\right)\right):=\lim _{\epsilon \longmapsto \infty} \frac{\log \left(B_{\epsilon}^{\beta}\left(\left(n_{k}\right)\right)\right.}{\log \epsilon}=\frac{H(X)}{-E(X) \log \beta},
$$

for $\mu$-almost all sequences $\left(n_{k}\right) \in \mathbb{N}^{\mathbb{N}}$.

Proof. Let $\left[n_{1}, n_{2}, \ldots, n_{k}\right]$ be a cylinder set in $\mathbb{N}^{\mathbb{N}}$. Applying the general ShannonMcMillan-Breiman theorem (see [2] and [4]) to the shift invariant measure $\mu$ with the countable partition $\{[n] \mid n \in \mathbb{N}\}$ of $\mathbb{N}^{\mathbb{N}}$, we have

$$
\lim _{k \longmapsto \infty}-\frac{1}{k} \mu\left(\left[n_{1}, n_{2}, \ldots, n_{k}\right]\right)=H(X)
$$

for $\mu$-almost all sequences of natural numbers $\left(n_{k}\right)$. Applying the law of large numbers we have

$$
\lim _{k \longmapsto \infty}-\frac{1}{k}\left|\left[n_{1}, n_{2}, \ldots, n_{k}\right]\right|_{\beta}=\lim _{k \longmapsto \infty}-\frac{1}{k} \beta^{n_{1}+n_{2}+\cdots+n_{k}}=-E(X) \log \beta
$$

for $\mu$-almost all sequences $\left(n_{k}\right)$. Hence

$$
\lim _{k \longmapsto \infty} \frac{\mu\left(\left[n_{1}, n_{2}, \ldots, n_{k}\right]\right)}{\left|\left[n_{1}, n_{2}, \ldots, n_{k}\right]\right|_{\beta}}=\frac{H(X)}{-E(X) \log \beta}
$$

for all sequences $\left(n_{k}\right) \in A_{\beta}$, where $A_{\beta}$ is a set of full measure; $\mu\left(A_{\beta}\right)=1$. Now fix a sequence $\left(n_{k}\right) \in A_{\beta}$. For all $\epsilon>0$ there is a $k>0$ with

$$
\left|\left[n_{1}, n_{2}, \ldots, n_{k}, n_{k+1}\right]\right|_{\beta}=\beta^{n_{1}+n_{2}+\cdots+n_{k}+n_{k+1}}<\epsilon \leq \beta^{n_{1}+n_{2}+\cdots+n_{k}}=\left|\left[n_{1}, n_{2}, \ldots, n_{k}\right]\right|_{\beta} .
$$

Since $B_{\beta^{n_{1}+n_{2}+\cdots+n_{k}}}^{\beta}$ is the cylinder $\left[n_{1}, \ldots, n_{k}\right]$, we have

$$
\left[n_{1}, \ldots, n_{k}, n_{k+1}\right] \subseteq B_{\epsilon}\left(\left(n_{k}\right)\right) \subseteq\left[n_{1}, \ldots, n_{k}\right]
$$

and thus

$$
\lim _{\epsilon \longmapsto \infty} \frac{\log \left(B_{\epsilon}^{\beta}\left(\left(n_{k}\right)\right)\right.}{\log \epsilon}=\frac{H(X)}{-E(X) \log \beta}
$$

for all sequences from the set of full measure $A_{\beta}$.

The Hausdorff dimension of a Borel measure $\nu$ on the real line is given by

$$
\operatorname{dim}_{H} \nu:=\inf \left\{\operatorname{dim}_{H} A \mid \nu(A)=1\right\}
$$

where $\operatorname{dim}_{H} A$ is the Hausdorff dimension of a set $A \subseteq \mathbb{R}$, see [16] or [5]. From Lemma 3.1 and Proposition 3.1, we obtain the following theorem using the local dimension of $\mu_{\beta}$.

Theorem 3.1 For the distribution $\mu_{\beta}$ of a power series with randomly increasing exponents $X_{\beta}$ we have

$$
\operatorname{dim}_{H} \mu_{\beta} \leq \frac{H(X)}{-E(X) \log \beta} .
$$


Proof. Let $A_{\beta}$ be the set defined in the proof of the last proposition and $C_{\beta}=\pi_{\beta}\left(A_{\beta}\right) \subseteq$ $\mathbb{R}$. We obviously have $\mu_{\beta}\left(C_{\beta}\right)=\mu\left(\pi_{\beta}^{-1}\left(\pi_{\beta}\left(A_{\beta}\right)\right)\right) \geq \mu\left(A_{\beta}\right)=1$. Now fix $x \in C_{\beta}$ and $\left(n_{k}\right) \in A_{\beta}$ with $\pi_{\beta}\left(\left(n_{k}\right)\right)=x$. By Lemma 3.1, $\pi_{\beta}$ is Lipschitz. Let $L$ be the Lipschitz constant. We have $B_{L^{-1} \epsilon}^{\beta}\left(\left(n_{k}\right)\right) \subseteq \pi_{\beta}^{-1}\left(B_{\epsilon}(x)\right)$ and hence

$$
\mu_{\beta}\left(B_{\epsilon}(x)\right)=\mu\left(\pi^{-1}\left(B_{\epsilon}(x)\right) \geq \mu\left(B_{L^{-1} \epsilon}^{\beta}\left(\left(n_{k}\right)\right)\right),\right.
$$

which implies

$$
\frac{\log \mu_{\beta}\left(B_{\epsilon}(x)\right)}{\log \epsilon} \leq \frac{\log \mu\left(B_{L^{-1} \epsilon}^{\beta}\left(\left(n_{k}\right)\right)\right)}{\log \epsilon} .
$$

Proposition 3.1 implies

$$
\limsup _{\epsilon \longmapsto 0} \frac{\log \mu_{\beta}\left(B_{\epsilon}(x)\right)}{\log \epsilon} \leq \frac{H(X)}{-E(X) \log \beta} .
$$

Now the local mass distribution principle, see [20] or [5], implies Theorem 3.1.

The singularity assertion in Theorem 2.1 is just a corollary to this result.

Corollary 3.1 If $\beta<e^{-H(X) / E(X)}$ the measure $\mu_{\beta}$ is singular with respect to the Lebesgue measure.

Proof. Under the assumption we have $\operatorname{dim}_{H} \mu_{\beta} \leq \frac{H(X)}{-E(X) \log \beta}<1$, which implies the singularity of $\mu_{\beta}$.

\section{Absolute continuity by transversality}

The following transversality result for the coding map $\pi_{\beta}$ is crucial to prove generic absolute continuity of the distribution $\mu_{\beta}$ of power series with randomly increasing exponents.

Proposition 4.1 For all $\bar{\beta} \in(0,1)$ there exists a constant $C>0$ such that for all sequences $\left(n_{k}\right),\left(m_{k}\right) \in \mathbb{N}^{\mathbb{N}}$

$$
\lambda\left\{\beta \in(\tilde{\beta}, 0.668)|| \pi_{\beta}\left(\left(n_{k}\right)\right)-\pi_{\beta}\left(\left(m_{k}\right)\right) \mid<\rho\right\}<C \rho \tilde{\beta}^{-\left(n_{1}+n_{2}+\cdots+n_{\mathbf{i}-1}+\min \left\{n_{\mathbf{i}}, m_{\mathbf{i}}\right\}\right)}
$$

for all $\rho>0$. Here $\mathbf{i}:=\min \left\{i \mid m_{i} \neq n_{i}\right\}$.

Proof. Assume without loss of generality that $m_{\mathbf{i}}>n_{\mathbf{i}}$. Consider

$$
\phi_{\left(n_{k}\right),\left(m_{k}\right)}(\beta):=\pi_{\beta}\left(\left(n_{k}\right)\right)-\pi_{\beta}\left(\left(m_{k}\right)\right)=\sum_{i=\mathbf{i}}^{\infty} \beta^{n_{1}+n_{2}+\cdots+n_{i}}-\beta^{m_{1}+m_{2}+\cdots+m_{i}}
$$




$$
\begin{gathered}
=\beta^{n_{1}+n_{2}+\cdots+n_{\mathbf{i}}}\left(1-\beta^{m_{\mathbf{i}}-n_{\mathbf{i}}}+\sum_{i=\mathbf{i}+1}^{\infty} \beta^{n_{\mathbf{i}+1}+\cdots+n_{i}}-\beta^{m_{\mathbf{i}}-n_{\mathbf{i}}} \beta^{m_{\mathbf{i}+1}+\cdots+m_{i}}\right) \\
=\beta^{n_{1}+n_{2}+\cdots+n_{\mathbf{i}}}\left(1+\sum_{i=1}^{\infty} a_{i} \beta^{i}\right)
\end{gathered}
$$

where $a_{i} \in\{1,0,-1\}$. Let $g(\beta):=1+\sum_{i=1}^{\infty} a_{i} \beta^{i}$. It is known that the smallest double zero of all power series that have this form is bigger than 0.668 , see [18]. Hence there is a constant $C>0$ independent of $g$ such that

$$
\lambda\{\beta \in(\tilde{\beta}, 0.668)|| g(\beta) \mid \leq \rho\} \leq C \rho,
$$

since all $g$ intersect horizontal lines near the axis transversally with bounded slope. Thus we obtain

$$
\lambda\left\{\beta \in(\tilde{\beta}, 0.668)|| \phi_{\left(n_{k}\right),\left(m_{k}\right)}(\beta) \mid \leq \rho\right\} \leq C \rho \tilde{\beta}^{-\left(n_{1}+n_{2}+\cdots+n_{\mathbf{i}}\right)},
$$

which is the claim of the proposition.

Now we are prepared to prove a generic result on the density of the measures $\mu_{\beta}$.

Theorem 4.1 For $q \in(1,2]$ define $\beta_{q} \in(0,1)$ by

$$
\beta_{q}=\sup \left\{\beta \mid\left(\sum_{n=1}^{\infty} \frac{p_{n}^{q}}{\beta^{n(q-1)}}\right)^{1 /(q-1)} \geq 1\right\} .
$$

For almost all $\beta \in\left(\beta_{q}, 0.668\right)$, the distribution $\mu_{\beta}$ of a power series with randomly increasing exponents $X_{\beta}$ is absolutely continuous with density in $L^{q}$.

Proof. Fix $\tilde{\beta}>\beta_{q}$. We consider the lower local density of the measure $\mu_{\beta}$ given by

$$
\underline{D}\left(\mu_{\beta}, x\right)=\liminf _{\rho \longmapsto 0} \frac{\mu\left(B_{\rho}(x)\right)}{2 \rho} .
$$

From [10] 2.12 we know that

$$
S:=\int_{\tilde{\beta}}^{0,0668} \int\left(\underline{D}\left(\mu_{\beta}, x\right)\right)^{q-1} d \mu_{\beta}(x) d \beta<\infty
$$

implies generic absolute continuity of $\mu_{\beta}$ with density in $L^{q}$ for the interval $(\tilde{\beta}, 0.668)$. Using standard arguments, see the proof of Theorem 4.1 in [15] or [11, 12], we obtain

$$
S \leq \liminf _{\rho \longmapsto 0}\left(\frac{1}{2 \rho}\right)^{q-1} \int\left(\int \lambda\left\{\beta \in(\tilde{\beta}, 0.668)|| \pi_{\beta}\left(\left(n_{k}\right)\right)-\pi_{\beta}\left(\left(m_{k}\right)\right) \mid<\rho\right\} d\left(m_{k}\right)\right)^{q-1} d\left(n_{k}\right) .
$$

Using Proposition 4.1 and integrating we have

$$
S \leq(2 C)^{q-1} \int\left(\int \tilde{\beta}^{-\left(n_{1}+n_{2}+\cdots+n_{\mathbf{i}-1}+\min \left\{n_{\mathbf{i}}, m_{\mathbf{i}}\right\}\right)} d\left(m_{k}\right)\right)^{q-1} d\left(n_{k}\right)
$$




$$
\begin{gathered}
\leq(2 C)^{q-1} \int\left(1+\sum_{i=1}^{\infty} \tilde{\beta}^{-\left(n_{1}+n_{2}+\cdots+n_{i-1}+n_{i}\right)} p_{n_{1}} p_{n_{2}} \cdots p_{n_{i}}\right)^{q-1} d\left(n_{k}\right) \\
\leq(2 C)^{q-1} \int\left(1+\sum_{i=1}^{\infty} \tilde{\beta}^{-(q-1)\left(n_{1}+n_{2}+\cdots+n_{i-1}+n_{i}\right)}\left(p_{n_{1}} p_{n_{2}} \cdots p_{n_{i}}\right)^{q-1} d\left(n_{k}\right)\right. \\
=(2 C)^{q-1}\left(1+\sum_{i=1}^{\infty} \sum_{n_{1}, \ldots, n_{i} \in \mathbb{N}^{i}} \tilde{\beta}^{-(q-1)\left(n_{1}+n_{2}+\cdots+n_{i-1}+n_{i}\right)}\left(p_{n_{1}} p_{n_{2}} \cdots p_{n_{i}}\right)^{q}\right) \\
=(2 C)^{q-1}\left(1+\sum_{i=1}^{\infty}\left(\sum_{n=1}^{\infty} \frac{p_{n}^{q}}{\tilde{\beta}^{n(q-1)}}\right)^{i}\right)<\infty
\end{gathered}
$$

since $\sum_{n=1}^{\infty} \frac{p_{n}^{q}}{\tilde{\beta}^{n(q-1)}}<1$ by the assumption $\tilde{\beta}>\beta_{q}$.

The assertion of absolute continuity in Theorem 2.1 is just a corollary to this result.

Corollary 4.1 For almost all $\beta \in\left(e^{-H(X) / E(X)}, 0.668\right)$ the measure $\mu_{\beta}$ is absolutely continuous.

Proof. Using de L'Hospital rule we have

$$
\lim _{q \longmapsto 1} \log \left(\left(\sum_{n=1}^{\infty} \frac{p_{n}^{q}}{\beta^{n(q-1)}}\right)^{1 /(q-1)}\right)=\sum_{n=1}^{\infty} p_{n} \log \left(p_{n} / \beta^{n}\right)=-H(X)-E(X) \log \beta .
$$

Hence

$$
\lim _{q \longmapsto 1} \beta_{q}=\sup \left\{\beta \mid e^{-H(X)-E(X) \log \beta} \geq 1\right\}=e^{-H(X) / E(X)} .
$$

This gives the result.

\section{References}

[1] M. Björklund and D. Schnellmann, Almost sure absolute continuity of Bernoulli convolutions, Ann. Inst. H. Poincaré Probab. Statist. 46 (3), 888-893, 2010.

[2] P. Billingsley, Ergodic Theory and Information, John Wiley and Sons, 1965.

[3] N. Bourbaki, Elements of Mathematics: General Topology, Addison-Wesley, 1966.

[4] K. Dajani and A. Fieldsteel, Equipartition of interval partitions and an application to number theory, Proc. Amer. Math. Soc. 129, 3453-3460, 2001.

[5] K. Falconer, Fractal Geometry - mathematical foundations and applications, John Wiley and Sons, 1990.

[6] A. Fan and J. Zhang, Absolute continuity of the distribution of some Markov geometric series, Science in China, Series A Mathematics, vol. 50, no.11, 1521-1528, 2007. 
[7] H. Fernau, Infinite Iterated function systems, Mathematische Nachrichten, vol. 170, issue 1, 79-91, 1994.

[8] J.E. Hutchinson, Fractals and self-similarity, Indiana Univ. Math. J. 30, 271-280, 1981.

[9] T. Jordan, M. Pollicott and K.Simon, Hausdorff dimension of randomly perturbed self-affine attractors, Comm. in Math. Phys. 270, 519-544, 2007.

[10] P. Mattila, Geometry of sets and measures in Euclidean spaces, Cambridge University Press, 1995.

[11] J. Neunhäuserer, Properties of some overlapping self-similar and some self-affine measures, Acta Mathematica Hungarica, vol. 92 (1-2), 143-161, 2001.

[12] J. Neunhäuserer, A general result on absolute continuity of non-uniform self-similar measures on the real line, Fractals, vol. 16, no. 4, 299-304, 2008.

[13] Y. Peres, W. Schlag and B. Solomyak, Sixty years of Bernoulli convolutions, Progress in Probability Vol. 46, Birkhauser, 39-65.

[14] Y. Peres and B. Solomyak, Absolutely continuous Bernoulli convolutions - a simple proof, Math. Research Letters 3, no. 2, 231-239, 1996

[15] Y. Peres and B. Solomyak, Self-similar measures and intersection of Cantor sets, Trans. Amer. Math. Soc 350, no. 10, 4065-4087, 1998

[16] Ya. Pesin, Dimension Theory in Dynamical Systems - Contemporary Views and Applications, University of Chicago Press, 1997.

[17] K. Simon and H. Toth, The absolute continuity of the distribution of random sums with digits $\{0,1, \ldots, m-1\}$, Real Analysis Exchange, vol 30(1), 397-410, 2005.

[18] P. Shmerkin and B. Solomyak, Zeros of $\{-1,0,1\}$ power series and connectedness loci of self-affine sets, Experiment. Math. 15, no. 4, 499-511, 2006.

[19] H. Toth, Infinite Bernoulli convolutions with different probabilities, Dis. Contin. Dyn. Sys. 21(2), 595-600, 2008.

[20] L.S. Young, Dimension, entropy and Lyapunov exponents, Ergod. Thy. \& Dynam. Sys. 2, 109-124, 1982. 